\title{
Gewalt in der Pflege
}

Monique Weissenberger-Leduc

Die Gewalt fängt nicht an, wenn Kranke getötet werden. Sie fängt an, wenn einer sagt: „Du bist krank: Du musst tun, was ich sage!" Aus: Die Gewalt von Erich Fried (in: Um Klarheit, 1985)

Grundbedürfnisse wie Wohlergehen, Freiheit, Selbstbestimmung und Identität sind absolut notwendige Bedingungen für das Leben. Decken wir diese nicht ab bzw. verhindern wir deren Befriedigung, üben wir Gewalt aus und sprechen den Anderen menschliche Würde ab. „Die Verhinderung der Befriedigung der Grundbedürfnisse ist Gewalt. " (Galtung 2007, 15)

"Alle Konflikte sind frei und gleich an Würde und Rechten geboren [...] ,Konfliktprävention' hat keine Bedeutung, aber ,Gewaltprävention“ hat eine wichtige Bedeutung und bringt allen Seiten Vorteile." (Galtung 2007, 9)

Auf der Grundlage von diesen Aussagen soll dieses Kapitel einen Beitrag zur Gewaltprävention in der Gestaltung des Alltags mit geriatrischen Patienten leisten. Jede im Sozialwesen beschäftigte Person ist aufgerufen, durch Fachwissen, Kompetenz, Fantasie und Kreativität Widersprüche zu überbrücken, die zu Sowohl-als-auch-Lösungen führen. Im Rahmen unserer Gedanken zur Gewaltverhinderung - sowohl an alten und hochaltrigen Menschen als auch an den Mitarbeiterinnen und Mitarbeitern einer Institution - versuchen wir einen Weg aufzuzeigen, der allen Beteiligten zugutekommt. 


\section{Was ist Gewalt?}

1992 hat Heinrich Popitz in seinem Werk Phänomene der Macht das immerwährende Phänomen der Gewalt sehr prägnant beschrieben:

„Der Mensch muss nie, kann aber immer gewaltsam handeln, er muss nie, kann aber immer töten - einzeln oder kollektiv - gemeinsam oder arbeitsteilig - in allen Situationen, kämpfend oder Feste feiernd - in verschiedenen Gemütszuständen, im Zorn, ohne Zorn, mit Lust, ohne Lust, schreiend oder schweigend (in Todesstille) - für alle denkbaren Zwecke - jedermann.“ (Popitz 1992, 50)

Johan Galtung, einer der Gründungsväter der Friedensforschung, weist schon 1975 darauf hin, dass es angesichts des Facettenreichtums von Gewalt nicht notwendig ist, die eine Definition des Begriffs zu finden. Vielmehr kommt es darauf an, theoretisch signifikante Dimensionen von Gewalt aufzuzeigen. In diesen Kontext muss der Gewaltbegriff einerseits weit genug definiert sein, um die breite Palette an Gewalt an alten und hochaltrigen Menschen zu erfassen. Anderseits muss die Definition spezifisch genug sein, um gewalttätige Handlungen und Unterlassungen klar zu verorten und gewaltverhindernde Maßnahmen beschreiben zu können.

\section{Formen der Gewalt an alten und hochaltrigen Menschen}

Der Begriff Gewalt (Elder Abuse; Elder Maltreatment, Neglect) ist kein klarer, eindeutig definierter Begriff. Er wird je nach Fachdisziplin unterschiedlich ausgelegt.

In der Toronto Declaration on the Global Prevention of Elder Abuse der Weltgesundheitsorganisation ist

„Gewalt an alten Menschen [...] eine einzelne oder wiederholte Handlung, oder das Fehlen einer angemessenen Handlung, die im Rahmen einer Beziehung geschieht, in der Vertrauen erwartet wird und die einer älteren Person Schaden oder Leid zufügt“, und „Gewalt an alten Menschen ist eine Menschenrechtsverletzung und eine signifikante Ursache von Verletzungen, Krankheit und Verzweiflung." (WHO 2002, Übersetzung Thomas Frühwald)

Mit dieser Definition wird der Aspekt der Enttäuschung einer Erwartungshaltung betont. Vertrauen in das Gegenüber - sie/er wird sich um mich 
kümmern, mich versorgen - macht das Gewalterlebnis nochmals schwerwiegender. Gleichzeitig bedeutet diese Einengung, dass Gewalt im öffentlichen Raum, zum Beispiel Raubüberfälle auf der Straße, ausgeschlossen wird. Hier wird also implizit schon auf die Pflegebedürftigkeit und die Betreuungspersonen Bezug genommen.

Das Phänomen der Gewalt an alten und hochaltrigen Menschen mit und ohne kognitive Beeinträchtigung (Demenz) ist nicht ortsgebunden und kann von professionellen Pflegepersonen wie von Angehörigen, aber auch von anderen alten Menschen ausgeübt werden. Gewalt erfährt man direkt durch eine bestimmte Person oder indirekt durch organisatorische Rahmenbedingungen einer Institution bzw. durch eine Gesellschaft, ihr Altersbild und ihre Regelungen für den Umgang mit alten Menschen; und schließlich umfasst Gewalt das äußerst weite Spektrum von Schlägen und Beschimpfungen über finanzielle Ausbeutung bis hin zur Überdosierung von Medikamenten, um nur einige wenige Beispiele zu nennen (Weissenberger-Leduc/Weiberg 2011). Gewaltanwendungen sind meist multidimensional.

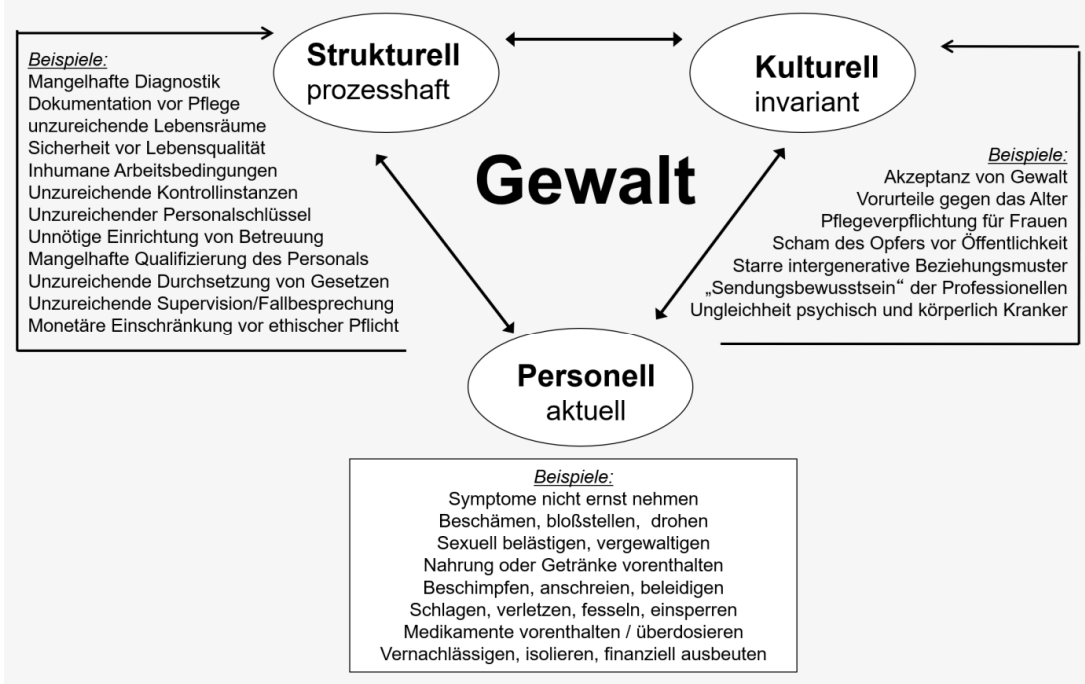

Abb. 1: Gewaltdreieck (Galtung 1993/Hirsch 2007) 


\subsection{Personelle, direkte Gewalt}

In Bezug auf die personelle, direkte Form der Gewalt gibt es verschiedene Versuche der weiteren Untergliederung. Eine sehr umfassende, aber auch praktikable Untergliederung wurde schon 1987 von Margret Dieck vorgenommen. Sie unterscheidet zunächst zwischen „Misshandlung“ (Abuse) und „Vernachlässigung“ (Neglect) (Dieck 1987, 311). Misshandlungen wiederum werden untergliedert in körperliche und psychische Misshandlung, finanzielle Ausbeutung sowie Einschränkung des freien Willens.

Vernachlässigungen zeigen sich als passive wie auch als aktive Vernachlässigungen (Dieck 1987, 311). Hier findet die Gewalt im sozialen Nahbereich von Familienkonstellationen statt. Sehr oft gibt es über Jahre TäterOpfer-Beziehungen im Rahmen eines einzigartigen Familiensystems. Besonders im Rahmen der personellen, direkten Gewalt sollte man sehr vorsichtig mit den Begriffen „Opfer“ und „Täter“ umgehen. Viele Opfer waren vorher Täter und vice versa.

Personelle, direkte Gewaltpotenziale finden sich auch in Organisationen des Gesundheitswesens (Krankenhäuser, Langzeitpflegeeinrichtungen, Tagesstätten und mobile Dienste). Laut Hirsch (2015) sind direkte personelle Gewalthandlungen oft Ausdruck von Hilflosigkeit, Scham, Ohnmacht, Überforderung, mangelhafter Unterstützung und Unkenntnis von Alternativen.

\subsection{Strukturelle, prozesshafte Gewalt}

Mit der strukturellen Gewalt kommen wir nun aber zu einer Form von Gewalt, die eine "Gewalt obne einen Akteur" (Galtung 1975, 12) ist. Hier wird keine direkte Gewalt ausgeübt, sondern es handelt sich um eine strukturimmanente Gewalt. Unter struktureller Gewalt kann man sowohl jene durch politische Strukturen hervorgerufene Gewalt verstehen als auch jene, die durch Strukturen einer Institution entsteht. Beispiele für strukturelle Gewalt an alten und hochaltrigen Menschen:

- unzureichende, den Bedürfnissen der geriatrischen Menschen nicht entsprechende Strukturen des Gesundheits- und Sozialwesens,

- erzwungenes Leben in institutionellen Rahmenbedingungen wegen nicht ausreichend vorhandener alternativer Betreuungsformen; Mangel an Privatsphäre und starre Tagesstruktur in Institutionen,

- zu kurze Aufenthaltsdauer in „nicht-geriatrisierten“ KrankenhausStrukturen, die zu einem Drehtüreffekt führen; nicht vorhandenes ger- 
iatrisches Assessment bei der Aufnahme oder nicht vorhandenes Entlassungs- bzw. Case-Management,

- Qualifikationsmangel - mangelnde Aus- und Weiterbildung - des Personals. In Österreich gibt es keine Facharztausbildung für Geriatrie. Es gibt nur eine Spezialisierung (ÖAK-Diplom), und die generalistische Ausbildung für den gehobenen Dienst in der Pflege (Diplom + Master in 4 Jahren) lässt keinen Platz für profundes geriatrisches Wissen.

- (versteckte) Rationierung, schlechte Personalressourcen; die Ökonomie überlagert und bestimmt Medizin und Pflege,

- mangelhafte Qualitätsstandards, unzureichende Kontrolle von Institutionen und 24-Stunden-Pflege,

- unangepasste Therapie, Pflege und Rehabilitation bzw. nicht ausreichende Rehabilitationskapazitäten für geriatrische Patienten,

- be- und verhinderndes Milieu: z. B. Delir, Frailty (Gebrechlichkeit), Inkontinenz, Sturz, Mangelernährung, Depression fördernd ...

In diesem Zusammenhang spricht Hörl von struktureller Gewalt, „wenn Menschen in eine Lage geraten, in der ihre aktuelle somatische und geistige Verwirklichung geringer ist als ibre potenzielle Verwirklichungschance" (Hörl 2014, 14). Hier gibt es keine gewaltvollen und gewaltfreien Phasen. Es handelt sich um eine permanente, statische Gewalt innerhalb einer sozialen Beziehung, die ausgesprochen nachhaltige negative Folgen auf die Lebensqualität, das Selbstwertgefühl und das Wohlbefinden des alten Menschen bewirken.

Neben dem (Nicht-)Vorhandensein eines konkreten Akteurs besteht ein weiterer Unterschied zwischen personeller und struktureller Gewalt in der Dynamik:

„Personale Gewalt steht für Veränderung und Dynamik - sie ist nicht nur eine sanfte Bewegung der Wellen, sondern bewegt selbst die sonst stillen Wasser. Strukturelle Gewalt ist geräuschlos, sie zeigt sich nicht sie ist im Grunde statisch, sie ist das stille Wasser." (Galtung 1975, 16)

Nimmt man diese beiden Aspekte zusammen - das Fehlen konkreter Akteure und die statische Kontinuität -, dann wird deutlich, dass im Fall der strukturellen Gewalt eine große Gefahr darin besteht, dass man sein eigenes Handeln durch sie rechtfertigt: „Die Bedingungen sind halt so", „Ich habe nicht die Zeit, mich mehr um die Bewohner zu kümmern" oder "Die Leitung will es so, was soll ich machen?" sind Beispiele für geläufige Erklärungsmuster - Erklärungsmuster für Handlungen oder Unterlassungen, die gewaltsam sind, aber entweder gar nicht mehr als gewalttätig wahrgenommen werden oder aber eben über die Rahmenbedingungen zu rechtfertigen ver- 
sucht werden. Man bekommt den Eindruck, dass Menschen, die im Rahmen solcher „repressiver Strukturen“ (Galtung 1975, 23) arbeiten, oftmals Abwehrstrategien entwickeln, wie etwa folgendes Beispiel zeigt:

„Weil der Ausdruck, freiheitsbeschränkend' oft durch den Ausdruck ,schützende Maßnahme' ersetzt wird, besteht die Gefahr, dass die wirkliche Anwendung von solchen Maßnahmen in der Pflegepraxis unterschätzt wird.“ (Hantikainen 2008, 3)

Einen besonderen Aspekt der strukturellen Gewalt im Gewalt-Dreieck von Galtung und Hirsch (siehe Abb. 1) möchte ich hier nur andeuten: „monetäre Einschränkung vor ethischer Pflicht“. Medizin/Pflege und Ökonomie oder Menschlichkeit und Effizienz sind keine Gegensätze. Es sind zwei unterschiedliche Logiken, die gemeinsam auf der Grundlage eines Dialogs in die Entscheidungen einfließen müssen. Der Begriff „Gesundheitswesen“ kann nicht durch den Begriff „Gesundheitsindustrie“ ersetzt werden. Es ist ein Widerspruch in sich, da das Gesundheitswesen ein Teil des Sozialsystems und die Gesundheitsindustrie ein Aspekt des Wirtschaftssystems ist.

Die Prioritäten des Sozialsystems wurden 2016 vom damaligen Sozialministerium ganz klar definiert:

„Das zentrale Interesse eines Sozial- oder Wohlfahrtstaates ist es, gesicherte Lebensbedingungen für alle Bürgerinnen/Bürger zu schaffen. Je nach Lebensphase oder Lebenssituation unterstützt der Sozialstaat Anspruchsberechtigte durch gezielte Leistungen, um ein selbstbestimmtes Leben zu ermöglichen." (Sozialministerium; Sozialstaat Österreich 2016, 14)

Die Prioritäten einer Gesundheitsindustrie basieren dagegen auf einem Produktionskonzept, das so viel Rendite wie möglich für die Investoren verspricht. Die Logik der Ökonomie muss im Dienst der Medizin und Pflege bleiben, nur so

- bleibt der Mensch als Patient/Subjekt im Zentrum einer individuellen Fürsorge.

- kann die Pflege ihre genuinen Aufgaben, die immer eingebettet in einer Patient-Umwelt-Beziehung sind und auf Kommunikation basieren, überhaupt wahrnehmen.

Die Aufgabe der Pflegeperson ist es, im Rahmen des Krankseins nicht nur einen Informed Consent zu erzielen (gemeinsame Erarbeitung der notwendigen Pflegemaßnahmen und -ziele), sondern vielmehr darauf zu achten, wo der Patient seine Selbstbestimmungsrechte im Alltag leben kann. 
Die größte Gefahr ist, dass die Ökonomie die Medizin und die Pflege überlagert und bestimmt. Dadurch wird die Pflege/Medizin sukzessiv in ihrer sozialen Praxis ausgehöhlt. In Anlehnung an Pierre Bourdieu wird Ökonomisierung als das Eindringen einer kapitalistischen Wirtschaftslogik in Teilsysteme - hier die Pflege - verstanden, die bis dahin eine eigene Logik hatten. Diese Ökonomisierung führt dazu, dass der Bedarf des Patienten nicht mehr allein reflektiert wird. Der Blick auf den Patienten als Mensch geht verloren und der Patient läuft Gefahr, funktionalisiert, ja in gewisser Weise verwertet zu werden. Dadurch verliert er seine Würde. Alles wird kontrolliert, muss evidenzbasiert sein, gemessen und belegt werden. Aber das ethisch qualifizierte Handeln der Pflegeperson besteht nicht allein in der Anwendung von Regeln, nicht nur im Messbaren, sondern vor allem in „der Qualität des Zuhörens, des In-Beziehung-Tretens, des SichEinlassen-Könnens, der Qualität der Begegnung mit dem kranken Menschen als Menschen“ (Maio 2016, 80). Folgende Aussage von Maio gilt genauso für das Pflegepersonal, das Gewaltprävention im Alltag leben möchte:

„Ärzte brauchen ganz offensichtlich immer mehr Mut, um genuin ärztlich zu entscheiden. Ein System aber, das den Ärzten ein schlechtes Gewissen bereitet, wenn sie sich für das Wobl des Patienten und nicht für die optimalen Erlöse der Klinik entscheiden, ist für die Medizin, deren Sinn und Zweck die Sorge um den Patienten ist, eindeutig das falsche." (Maio 2016, 35)

Welche Folgen können Fragmentierung und Ziffern im Kopf des Pflegepersonals haben?

Viele Pflegende verweisen in den verschiedenen Befragungen darauf, dass u. a. die mobilisierende Pflege, d.h. das Bemühen, den Patienten wieder auf die Beine zu bringen, oft nicht mehr geleistet werden kann, bzw. verliert ein Patient zum Beispiel aufgrund einer gelungenen Pflege im Tageszentrum die Pflegestufe eins, darf er nicht mehr ins Tageszentrum gehen. Die Kosten werden nicht mehr übernommen.

Viele Kommentare belegen, dass Pflegepersonen sich immer weniger Zeit nehmen, um Patienten durch zwischenmenschliche Gespräche, durch Hilfestellungen, durch Beratungen und Schulungen auf die Entlassung vorzubereiten.

Die fürsorgende und empathische Beziehung wird durch den Standard der Unpersönlichkeit ersetzt. Die Betreuung und Behandlung wird auf eine technische Reparatur reduziert. Alles ist bestens dokumentiert und kodiert. Aber der Mensch als Patient wird mit seiner Befindlichkeit und der psychosozialen Dimension des Krankseins allein gelassen. 
Eine Vertiefung dieser Problematik hat die Ethikkommission beim Bundeskanzleramt 2018 in der Stellungnahme Medizin und Ökonomie vorgenommen.

\subsection{Kulturelle, invariante Gewalt}

Mit der Betrachtung kultureller Gewalt wird die Gruppe der Gewalt ausübenden und Gewalt erleidenden Personen nochmals vergrößert. Ist die personelle Gewalt auf einzelne Personen oder Personengruppen als Akteure beschränkt, sind im Rahmen von struktureller Gewalt doch schon weit mehr Menschen als ausübende (Mit-)Täter beteiligt, in unserem Fall also all jene Menschen, die in irgendeiner Form mit der Betreuung von alten und hochaltrigen Menschen zu tun haben - seien sie nun Politiker oder andere Personen, die entsprechende Gesetze oder Verordnungen erlassen, seien sie Leitungspersonen eines Pflegeheims, die sich weder für die Arbeitsbedingungen der Mitarbeiter noch für die Lebensbedingungen der Bewohner sonderlich engagieren, seien sie Ärzte, die eine genaue Diagnose und Behandlung im Fall von geriatrischen Menschen mit kognitiver Beeinträchtigung als zu zeitaufwendig oder gar als Zeitverschwendung ansehen, seien sie Angehörige, die gewalttätige Handlungen bzw. Unterlassungen ihrem alten und hochaltrigen Familienmitglied gegenüber tolerieren (oder selbst Gewalt ausüben).

Kulturelle Gewalt ist etwas, das jedes Mitglied der Gesellschaft betrifft, denn hier geht es vor allem um Stereotype und Vorurteile, um unreflektierte Wertungen von (zum Teil großen) Teilen einer Gemeinschaft - mit gravierenden Konsequenzen für die „Verurteilten“. Kulturelle Gewalt ist weder auf bestimmte Personengruppen beschränkt noch gibt es bestimmte klar zu identifizierende Orte, an denen sie vermehrt zu beobachten ist.

Mit Johan Galtung halten wir fest, dass kulturelle Gewalt gewissermaßen den grundsätzlichen Kontext bietet, vor dessen Hintergrund gewalttätige Handlungen und Unterlassungen (personeller wie struktureller Natur) legitimiert werden und nicht mehr als solche erscheinen - man nimmt sie nicht als Unrecht wahr.

„Kulturelle Gewalt funktioniert zum einen dadurch, daß sie die ,moralische Färbung' einer Handlung von rot/falsch auf grün/richtig oder zumindest auf gelb/akzeptabel schaltet; ein Beispiel hierfür wäre: ,Töten im Namen eines Landes ist gerechtfertigt, im eigenen Namen jedoch ungerechtfertigt ${ }^{*}$. Andererseits macht sie die Realität so undurchsichtig, daß wir eine gewalttätige Handlung oder Tatsache überhaupt 
nicht wahrnehmen oder sie zumindest nicht als solche erkennen.“ (Galtung 1993, 106)

Galtung weist in diesem Zusammenhang darauf hin, dass wir in verschiedensten Bereichen einer Kultur Elemente kultureller Gewalt vorfinden (seien es bestimmte Aspekte einer Religion, seien es Aspekte unserer Sprache, aber auch unserer Wissenschaft). In all diesen Bereichen treffen wir auf Vorurteile, auf Wertungen, die in Zusammenhang stehen mit Dichotomien wie Selbst und Anderer, Eigenes und Fremdes, Erhöhung und Erniedrigung (Galtung 1993, 109). Man denke hier etwa an Dichotomien wie Gläubiger - Ungläubiger, Inländer - Ausländer, Jung - Alt, Leistungswillige „Schmarotzer“, Rationalität - Irrationalität ... Rolf Hirsch nennt folgende Beispiele für kulturelle Gewalt in Bezug auf alte Menschen:

- Pflegeverpflichtung für Frauen

- Scham der Opfer vor Öffentlichkeit

- starre intergenerative Beziehungsmuster

- „Sendungsbewusstsein“ der Professionellen

- Ungleichheit psychisch und körperlich Kranker (Hirsch 2001, 4)

- Akzeptanz von Gewalt, wie zum Beispiel:

- Die Patientin MUSS essen.

- Die Patientin MUSS fixiert werden.

- Die Patientin IST dement.

- Durch ein "autoritäres Auftreten" bringt man die Patientin schon zum Essen.

- Reden bringt da nichts, da hilft nur Haldol.

- Wenn ich freundlich bin, erwarte ich mir das auch vom Patienten.

- Go-Goes, Slow-Goes, No-Goes ${ }^{2}$

Gewalthandlungen entstehen oft auf dem Boden von Vorurteilen, Stereotypen, Irrglaube und Mythen. Gemeinsam ist Vorurteilen und Stereotypen, dass sie sich auf soziale Gruppen beziehen. Menschen werden hierbei aufgrund eines bestimmten Kriteriums wie Geschlecht, Alter oder Nationalität einer sozialen Gruppe zugeordnet. Bei Vorurteilen geht es um die Verknüpfung einer sozialen Gruppe mit mehrheitlich negativen Attribu-

1 Haloperidol - ein Neuroleptikum, das zur Behandlung schizophrener Symptome eingesetzt wird

2 eine in Amerika gängige Unterteilung von älteren Menschen in Go-Goes, die noch mobil sind und die Welt erkunden, sogenannte Slow-Goes, die nicht mehr so aktiv sind und die meiste Zeit zu Hause verbringen, und No-Goes, die sich nicht mehr allein bewegen können und auf Hilfe angewiesen sind 
ten (die ebenfalls für diese Gruppe als typisch angesehen werden) und mit affektiven Reaktionen, also mit Emotionen wie Hass, Furcht, Neid etc.

Beispiele für Vorurteile gegen alte Menschen, besonders wenn sie kognitive Beeinträchtigungen haben:

- ... hört nicht $z u, \ldots$ versteht nichts

- ... IST unbelehrbar, ... IST stur

- ... schreit dauernd

- ... IST mühsam

- ... IST aggressiv, ... IST bösartig

Wenn wir „ist“ sagen, dann geben wir einen unlöschbaren Stempel auf die Stirn des „Verurteilten“.

Besonders problematisch ist eine spezifische Art von kultureller Gewalt: Wenn beispielsweise eine Pflegeperson die gängigen Vorurteile gegenüber dem Alter teilt und davon ausgeht, dass alte Menschen sowieso senil und gleichzeitig halsstarrig seien, wird der alte Mensch, der von ihr betreut wird, oftmals in seiner Selbstbestimmung eingeschränkt werden - und eventuell wird die Pflegeperson diese Handlungen nicht einmal als gewalttätig einstufen.

Bei Stereotypen hingegen handelt es sich um Vorstellungen über eine Gruppe, die nicht automatisch mit Wertungen verbunden sein müssen. So ist das Stereotyp „alte Menschen sind gebrechlich“ eine Überzeugung, die nicht unbedingt mit irgendeiner Emotion verknüpft sein muss (aber natürlich leicht verknüpft werden kann). Es geht also zunächst schlicht um die Verknüpfung einer sozialen Gruppe mit bestimmten, für sie als typisch angesehenen, verallgemeinerten Eigenschaften.

Es gibt eine Vielfalt an biologischen, sozialen, kulturellen, ökonomischen und milieubedingten Faktoren, die das Risiko erhöhen. Besonders betroffen sind vor allem ältere, pflegebedürftige, multimorbide und in Armut lebende Personen sowie Menschen mit körperlichen und kognitiven Einschränkungen (Demenz), die auf Hilfe Dritter angewiesen sind.

Zusammenfassend schließen wir uns der Meinung von Galtung an. Wir verstehen Gewalt als eine „vermeidbare Beeinträchtigung menschlicher Grundbedürfnisse“ (Galtung 1993, 106), die entweder direkt von einem oder mehreren Akteuren oder aber strukturell bzw. kulturell ausgeübt wird und die beim Opfer Schaden und/oder Leid hervorruft. 


\section{Gewalt an alten Menschen - Prävalenz}

Die Inzidenz- und/oder Prävalenzzahlen für die Diagnose „Gewalt an alten Menschen" sind nicht eindeutig, da vieles nicht ans Tageslicht kommt. In Österreich gibt es bis jetzt keine Opferstudien und dadurch kein „brauchbares Datenmaterial“ (Ogris 2017, 15). Ogris schreibt weiter: Es „gibt eine besonders hohe Diskrepanz zwischen Prävalenz, Anzeigen und Verurteilungen" (Ogris 2017, 17). Wir wissen nur, dass Gewaltanwendung gegen alte Menschen weltweit stattfindet, und „,[m]indestens vier Mio. Menschen werden jedes Jahr in Europa misshandelt und ca. 2500 von ihnen sterben daran" (WHO 2011, Abstract).

Die Dunkelziffer („das Verhältnis zwischen der Zahl der statistisch ausgewiesenen und der wirklich begangenen Straftaten“; Pflegerl/Cizek 2002, 63) der Gewaltanwendungen ist wahrscheinlich sehr hoch und es ist anzunehmen, dass sich in den letzten zehn Jahren nichts geändert hat, vergleichen wir die Daten von Cooper et al. von 2008 mit den aktuellen Daten von Nursing Home Abuse Center Protecting Our Seniors: Elder Abuse Statistics von 2018.

Cooper et al. führten 2008 eine systematische Literaturrecherche durch. Sie konnten im Rahmen der Untersuchung von 49 Studien nur sieben finden, die den wissenschaftlichen Kriterien von Erkennungs- und Messmethoden entsprachen. Die Ergebnisse können folgendermaßen zusammengefasst werden:

- 6,3 Prozent der Personen im Alter von über 65 Jahren erlebten eine signifikante Gewalterfahrung im vergangenen Monat und 5,6 Prozent der in Partnerschaften lebenden Menschen (über 65 Jahren) gaben die Anwendung von körperlicher Gewalt im letzten Jahr an;

- 5 Prozent der pflegenden Angehörigen berichteten über körperliche Gewaltanwendung im letzten Jahr im Zusammenhang mit der Betreuung der pflegebedürftigen und an Demenz erkrankten alten Menschen. Ein Drittel berichtete über irgendeine andere Form von Gewaltanwendung;

- 16 Prozent des Pflegeheimpersonals gaben zu, signifikante psychische Gewalt angewendet zu haben;

- nur ein bis zwei Prozent der Fälle von Gewaltanwendung wurden an die Heimleitung weitergeleitet. (Cooper et al. 2008)

Hirsch meinte am 09.06.2016 bei der 6. Fachtagung Trauma-Netzwerk Niedersachsen, in der Bundesrepublik Deutschland haben „25 Prozent der 60- bis 84-Jährigen [hier ist die gesamte Bevölkerung zwischen 60 und 84 
gemeint; Anm. M. W.-L.] [...] innerhalb eines Jahres Gewalterfahrungen machen müssen“.

Die aktuellen Daten von Nursing Home Abuse Center Protecting Our Seniors: Elder Abuse Statistics von 2018 zeigen ein ähnliches Bild:

"According to the National Committee For The Prevention Of Elder Abuse, about 5 percent of the older population has suffered from some kind of abuse.

A study of 2,000 nursing facility residents indicated an abuse rate of 44 percent and a neglect rate of 95 percent.

Complaints of abuse, exploitation or neglect accounted for 7 percent of complaints given to Ombudsmen at long-term care facilities.”

(Traxler 2018, Quick Answer)

Damals wie jetzt kommt nur die Spitze des Eisbergs ans Tageslicht; die aktuellen Daten diesbezüglich für die USA sprechen eine klare Sprache:

"According to the best estimates, about 1-2 million U.S. citizens 65 years of age or older have been mistreated, exploited or injured by a caregiver. Only about one out of every 14 incidents of elder abuse (including selfneglect) in domestic settings actually come to the attention of local or state authorities.

Only one out of every 25 cases of financial exploitation are reported. These unreported incidents would increase the amount to 5 million victims of financial exploitation per year.

Another study out of the National Elder Abuse Incidence Study indicated that about 20 percent of cases of neglect, exploitation, abuse or self-neglect are reported.” (Traxler 2018, Quick Answer)

Es scheint einen Zusammenhang zu geben zwischen Vernachlässigung und kognitiver Beeinträchtigung bzw. Ausmaß des funktionellen Defizites (Jackson 2012). Genaue Zahlen für Gewalt an Menschen mit kognitiven Beeinträchtigung gibt nur die Nursing Home Abuse Center Protecting Our Seniors: Elder Abuse Statistics von 2018: „According to research, dementia is a risk factor for abuse. A study showed that 47 percent of patients with dementia suffered from some kind of abuse" (Traxler 2018, Quick Answer).

47 Prozent! Das bedeutet, fast jeder zweite Mensch mit kognitiver Beeinträchtigung erfährt irgendeine Art von Gewalt! Es sind viel mehr als die 25 Prozent, die der Geriater Thomas Frühwald in der Gruppe der älteren multimorbiden Menschen, die in ihrer Bewegungsmöglichkeit einge- 
schränkt und oft an Demenz erkrankt sind, 2008 feststellte. Die Zahl entspricht aber den Erkenntnissen von Görgen:

„Psychische Misshandlung Pflegebedürftiger durch ihre pflegenden Angehörigen ist in der untersuchten Stichprobe mit einer 12-MonatePrävalenz von $47.6 \%$ am weitesten verbreitet. [...] Formen physischer Misshandlung berichten für einen Zeitraum von 12 Monaten 19.4\% der Befragten." (Görgen et al. 2012, 33)

Gleichzeitig wissen wir, dass Gewalt an alten Menschen im Rahmen von Pflegebeziehungen meist schattenhaft bleibt. Nur die gravierenden Gewaltanwendungen, deren Folgen für Dritte sichtbar sind, werden weitergeleitet bzw. zur Anzeige gebracht. Wenn der alte Mensch Gewalt erfährt, weiß er nicht, an wen und in welcher Weise er sich an jemanden wenden soll, nicht zuletzt, da er von den Pflegenden abhängig ist. Wer entscheidet über eine eventuelle Anzeige? (Weissenberger-Leduc/Weiberg 2011).

\section{Folgen von Gewalt an alten Menschen}

Die Folgen von Gewalt sind verheerend: Alte Menschen, die Gewalt erfahren haben, haben ein um 300 Prozent erhöhtes Risiko für Mortalität, chronische Erkrankungen, inklusive Depression, Angst, chronische Schmerzen, Hypertonie und Herz-Kreislauf-Probleme als Menschen über 65, die keine Gewalt erfahren haben (Cannell 2016). Schon Lachs machte 1998 auf die Gewaltfolgen bei geriatrischen Patienten aufmerksam:

- unmittelbare Verletzungsfolgen bei physischer Gewalt;

- Vernachlässigungsfolgen sind u. a. Exsikkose, Malnutrition, Dekubitus, Kontrakturen, Inkontinenz ...

- Depression, Angst, Regression, soziale Isolation und dadurch beschleunigter kognitiver Abbau;

- Mechanische Fixierungen führen zu Immobilisierung, Dekonditionierung und höherer Sturz- und Verletzungsgefahr.

- „Chemische“ Fixierung führt zu Polypharmazie mit multiplen Nebenwirkungen, kognitivem Abbau, Stürze, Malnutrition ...

- mehr Pflege- und Betreuungsbedarf, höhere Mortalität; Dreijahresmortalität: 91 Prozent bei Gewaltopfern vs. 58 Prozent ohne Gewaltanwendung (Lachs 1998);

- Der vermehrte Pflege- und Betreuungsbedarf schließt den Kreislauf ab, da es zu erhöhtem Betreuerstress, Abstumpfung und Potenzierung des Burnouts kommt. 


\section{Gesundheitsfördernde und präventive Interventionen, Maßnahmen und Ansätze im thematischen Kontext}

Artikel 3 der Europäischen Menschenrechtskonvention (EMRK, in Kraft getreten am 3. September 1953) ist eindeutig: „Niemand darf der Folter oder unmenschlicher oder erniedrigender Strafe oder Behandlung unterworfen werden." Diese internationale Verpflichtung zum Gewaltschutz hat Österreich ratifiziert, genauso wie die Istanbul-Konvention, die am 1. August 2014 in Kraft getreten ist.

Schon 2002 klassifizierte die WHO in der Toronto-Deklaration die Maßnahmen zur Gewaltprävention auf zwei Ebenen. Die zeitliche Ebene beinhaltet die primäre, sekundäre und tertiäre Gewaltprävention. Die Zielgruppenebene beschäftigt sich mit Strategien, die sich allgemein an jedermann wenden, aber auch mit Interventionen, die sich speziell an Täter und Opfer oder an „Hochrisikogruppen“ richten.

\subsection{Primäre, sekundäre und tertiäre Prävention}

Strategien der Gewaltprävention lassen sich - wie die Strategien der Krankheitsprävention - je nach Zeitpunkt der Intervention in primäre, sekundäre und tertiäre Schritte unterscheiden. Diese Interventionen verfolgen das Ziel, in den Prozess der Gewalt einzugreifen. So kann dieser Prozess schon im Vorfeld oder im Frühstadium vorbeugend abgebrochen, in seiner Dynamik gemindert oder rückgängig gemacht werden.

\subsubsection{Primäre Prävention}

Die primäre Prävention richtet sich darauf, im Vorfeld einer Gewaltentwicklung die Widerstandskräfte zu stärken, das Auftreten einer Gewalt möglichst ganz zu vermeiden oder doch zumindest die Verbreitung einer Gewalt so niedrig wie möglich zu halten. Primärprävention kann unspezifisch (generell) orientiert sein und die gesamte Bevölkerung ansprechen. Sie kann aber auch spezifisch sein und sich gezielt an Menschen richten, die ein erhöhtes Risiko für Gewaltanwendungen tragen. Beispiele für Initiativen der Primärprävention sind Vorlesungen (wie „Eine von fünf“ an der medizinischen Universität Wien), Sensibilisierungskampagnen, Bewusstseinsbildungsprozesse, Broschüren (wie „Gewalt erkennen“, „Breaking the Taboo“, „Mind the Gap“ oder die Bonner Initiative gegen Gewalt 
im Alter „Handeln statt Misshandeln“ ...), Seminare, Workshops für Führungspersonen des Managements und Mitarbeiter in Sozialbetreuung und/ oder in Betreuungs- und Pflegeorganisationen, Vermittlung gewaltbezogenen Wissens in Form von „One-off"-Broschüren, Videos, Internet, Stationsbibliothek, Ausstellungen, Gewaltsprechtage, Informationsveranstaltungen zur Gewaltprävention etc.

\subsubsection{Sekundäre Prävention}

Die sekundäre Prävention umfasst alle Maßnahmen zur Entdeckung von Gewalt und ihre erfolgreiche Früherkennung. Die wichtigste Aufgabe ist das frühe und schnelle Handeln, um die weitere Entfaltung von Gewalt und/oder Gewaltkomplikationen zu vermeiden. Eine wichtige Rolle spielt in Österreich $\$ 8$ (5) des Bundesgesetzes über Krankenanstalten und Kuranstalten: „Den Opferschutzgruppen obliegen insbesondere die Früherkennung von häuslicher Gewalt und die Sensibilisierung der in Betracht kommenden Berufsgruppen für häusliche Gewalt.“

\subsubsection{Tertiäre Prävention}

Die tertiäre Prävention zielt darauf ab, die Schwere einer Gewalttat und ihre Wiederholung zu verhindern. Sie ist auf Menschen gerichtet, die bereits Gewalt verursacht und/oder erfahren haben. Hier geht es hauptsächlich um Verhaltensprävention und Verhältnisprävention.

Tertiäre Präventionsmaßnahmen beziehen sich im Bereich der Verhaltensprävention auf das Verhalten von Individuen und Gruppen bei und im Zusammenhang mit der Gewalt. Zu den Maßnahmen zur Verhaltensprävention gehören insbesondere personenbezogene Ansätze wie Anti-Aggressionstraining, Informations- und Aufklärungsmaßnahmen, Maßnahmen zur Vermittlung von Bewältigungstechniken zur Verhaltensänderung, soziale Kompetenzen, Selbstkontrolle sowie soziale Informationsverarbeitung. Da Gewalt an geriatrischen Menschen mit und ohne kognitive Beeinträchtigung sehr oft in der Familie stattfindet, müssen auch familienbezogene Ansätze stattfinden: Förderung von gewaltmindernden familiären Strukturen, Unterstützung bei der Suche von Gewaltpotenzialen, familienzentrierte Lösung, konsistente, akzeptierende, person- und ressourcenorientierte und fördernde Betreuung und Pflege, positive Konfliktbearbeitung in der Familie. 
Im Bereich der Verhältnisprävention steht die Veränderung der biologischen, sozialen Umwelt im Mittelpunkt. Hier gibt es zwei verschiedene Ansätze:

Bei Gewalt im erweiterten Familienkreis müssen einerseits Maßnahmen gesetzt werden, die Gewalt verhindern: Entlastung des pflegenden Angehörigen durch Sozial- und Besuchsdienste, Aufnahme im Tageszentrum oder im Pflegeheim. Spätestens jetzt soll eine Sozialarbeiterin in die weitere Betreuung integriert werden und die Frage gestellt werden: Welche Ressourcen stehen grundsätzlich zur Verfügung?

Menschen mit kognitiver Beeinträchtigung und ihre Nahestehenden sind im Laufe der Krankheit mit großen Veränderungen, Belastungen und Herausforderungen konfrontiert. Anstatt pauschale Voraussagen über z.B. phasenspezifische Bedürfnisse als Richtschnur für Interventionen zu machen, ist ein individuelles geriatrisches Assessment der konkreten Situation, auch mit Hilfe von wissenschaftlich getesteten Instrumenten (z. B. zur Erfassung der Belastung der pflegenden Angehörigen oder der Ressourcen im Umfeld), zu empfehlen (Schiffczyk et al. 2013).

Bei Gewalt in Organisationen andererseits ist es wichtig, das Problem des Elder Abuse achtsam wahrzunehmen und eine Grundhaltung der Unterstützung einzunehmen.

Die drei Ebenen der Prävention werden durch die zentralen fünf „P“ der Istanbul-Konvention klar definiert:

- Primäre Prävention:

- Policy - eine entsprechende Politik

- Prevention - Maßnahmen der Prävention

- Sekundäre Prävention:

- Provision - angemessene Hilfsangebote

- Protection - Schutz für gefährdete Personen

- Tertiäre Prävention:

- „Strafverfolgung von Tätern, um gefährdete Personen aktiv vor Gewalt zu schützen." (Nachbaur 2017, 41)

Damit haben alle Mitarbeiter im Gesundheitswesen als Privatperson und aufgrund ihres Berufes seit 1953 einen klaren, verpflichtenden Auftrag: Gewalt jeder Art zu vermeiden.

Aber würden wir die Gewalt wahrnehmen, müssten wir etwas tun: Verantwortung übernehmen. Es könnte sehr unbequem werden, so verschließen wir die Augen vor der Wahrheit. Deswegen ist - wie Anselm Grün meinte - Gleichgültigkeit Gewalt. Wir müssen hinschauen, das Schweigen brechen. Professionelle und Selbstverantwortung sind gefragt. Die Miss- 
handlung alter Menschen darf nicht mehr ein Tabu sein. Salutogenetisch gesehen bedeutet dies für mich als Mitarbeiterin im Gesundheitswesen, besonders in der geriatrischen Pflege, und für mich persönlich als Privatperson:

- Ich sehe, erkenne und verstehe die Problematik der Gewalt.

- Ich kann etwas tun, proaktiv kompetent gestalten.

- Der Kampf gegen Gewalt ist Teil meiner Pflege- und Lebensphilosophie.

Andreas Kruse macht uns auf eine Grundvoraussetzung für einen „gewaltfreien" Umgang mit Menschen mit Demenz aufmerksam:

„Die Begegnung mit einem demenzkranken Menschen stellt die Kontaktperson vor eine bedeutende psychologische Aufgabe - nämlich vor die Aufgabe der Auseinandersetzung mit sich selbst, mit den möglichen Begrenzungen des eigenen Lebens. In dem Maße, in dem es gelingt, diese Auseinandersetzung in einer persönlich konstruktiven Weise zu leisten, wird auch die Grundlage für wirkliche Begegnung geschaffen, die eine Voraussetzung für eine fachliche wie ethisch anspruchsvolle Therapie und Pflege bildet." (Kruse 2008, 50)

Der WHO-Bericht Dementia: a Public Health Priority von 2012 beleuchtet die biologischen, sozialen, kulturellen, ökonomischen und milieubedingten Faktoren, die das Risiko erhöhen, Opfer oder Täter in der Misshandlung eines älteren Menschen zu werden, sowie Schutzfaktoren, die ebendies verhindern helfen. Es liegen einige Erkenntnisse über wirksame Interventionen vor, hierunter Programme für eine psychologische Beeinflussung potenzieller Täter und für eine Haltungsänderung gegenüber älteren Menschen, für ein besseres psychisches Wohlbefinden der Betreuer und für das Erlernen von Beziehungsfähigkeit und sozialen Kompetenzen schon in jüngeren Jahren. Die Erkenntnisgrundlage muss noch erweitert werden, doch zeigen Befragungen, dass Öffentlichkeit und Politik die Problematik an sich erkannt haben. Der Bericht regt eine Reihe von Maßnahmen an, welche Mitgliedstaaten, internationale Organisationen, nichtstaatliche Organisationen, Forscher, Praktiker und andere Akteure ergreifen könnten, um eine bessere konzeptionelle Antwort zu ermöglichen und angemessene Mittel für das Thema zu mobilisieren.

2016 erschien eine Cochrane Database of Systematic Reviews mit dem Titel Interventions for preventing abuse in the elderly (review) (Baker et al.). Diese systematische Literaturrecherche dient als wissenschaftliche Grundlage, obwohl Baker et al. eindeutig zeigen, dass die meisten Studien leider nur 
von schwacher bzw. sehr schwacher Qualität sind. Hier muss nicht nur weiter geforscht werden, sondern die Qualität der Studien muss zunehmen. Dies ist notwendig, um eine bessere Evidenz der Wirkung zu erreichen und damit die knapp verfügbaren Ressourcen optimal einzusetzen. Baker et al. nennen vier Hauptinput-Gruppen - Government planners, Community based groups, Institutions and Legislators -, die drei klar definierte Ziele verfolgen: "to avoid potential elder abuse, to lower rates of elder abuse in communities and in institutions and to reduce recurrent elder abuse" (Baker et al.).

\subsection{Warnzeichen für Gewalt an älteren Menschen}

Wann man bei der Anamnese hellhörig werden sollte:

- bei inkohärenten, widersprüchlichen Erklärungen für festgestellte Verletzungen,

- wenn die Betreuungsperson Überforderungs- bzw. Erschöpfungszeichen zeigt,

- bei lautem, verbal aggressivem Ton zwischen Betreuer und betreuter Person in

- Anwesenheit anderer,

- wenn die betroffene ältere Person depressiv, verängstigt oder verschreckt erscheint,

- wenn der alte Mensch ungepflegt ist, die Wohnung ebenfalls,

- beim Fehlen von Habseligkeiten und Geld der betroffenen Person,

- wenn die ältere Person daran gehindert wird, mit Ärzten oder Pflegepersonen allein zu sprechen,

- beim Meiden von Arztvisiten oder Besuchen der Sozialdienste,

- bei vielen unbezahlten Rechnungen und

- bei einem leeren Kühlschrank und einer kalten Wohnung. (Frühwald 2008)

Ein Gewalt-Screening verlangt:

- hohes Problembewusstsein und gutes Urteilsvermögen,

- ungestörtes Gespräch mit der alten Person,

- einfühlsame Fragen und Beurteilung im Hinblick auf:

- physische Gewaltanwendung,

- aktive/passive Vernachlässigung,

- psychische Gewalt und 
- Betreuerstress, Burnout,

- Selbstvernachlässigung, „self-neglect“ (DD.: Messie-Syndrom),

- Berücksichtigung ethnischer, kultureller Unterschiede,

- Beurteilung der kognitiven Fähigkeit/Möglichkeit der Betroffenen, selbst über die Situation zu urteilen und zu entscheiden, sowie

- Respektierung der Autonomie der älteren Person. (Frühwald 2008; Lachs 2015)

Beispiele für Fragen bei der Anamneseerhebung bei Verdacht auf Gewalt:

- Möchten Sie mir etwas über schwierige, Sie belastende Situationen oder unangenehme Zwischenfälle erzählen?

- Hat jemand versucht, Sie zu verletzen?

- Wurden Sie angebrüllt, beschimpft?

- Wurden Sie gezwungen, Dinge zu machen, die Sie nicht wollten?

- Kommen Sie mit Ihrem Geld aus?

- Haben Sie etwas unterschreiben müssen, ohne zu wissen, was es ist?

- Fürchten Sie sich vor jemandem?

- Werden Sie oft alleine/ohne Hilfe gelassen?

- Sind Ihnen Sachen abhandengekommen? (Frühwald 2008)

\subsection{Gesundheitsfördernde und präventive Interventionen, Maßnahmen und Ansätze im thematischen Kontext}

Wenn Führungskräfte Mitarbeitern mehr Freiheit und Wahlmöglichkeiten geben wollen, müssen sie mit ihnen Kompetenzfähigkeiten entwickeln und trainieren - nur so erreichen Mitarbeiter einen höheren Grad an Selbstverantwortung im Hinblick darauf, die humanistischen, gewaltarmen Entwicklungen besser zu verstehen und mitzutragen (Bauer 2014). Eine humanistische, gewaltarme Haltung kann nicht aus Büchern oder Ratgebern gelernt werden, sondern sie wächst als Ausdruck einer inneren Einstellung. Anstatt nur Menschen durch die Brille der Defizite, der Ablehnung, der Abwehr, des Ekels zu sehen, versuchen dann Mitarbeiter immer wieder, in der Begegnung mit multimorbiden Menschen ,irgendetwas $z$ u entdecken, das sie mögen und das ibnen gefällt" (Hüther 2017, 84). Solche Mitarbeiter können dann multimorbiden Menschen mit Respekt von Subjekt zu Subjekt begegnen und gemeinsam in Beziehung werden sie ein Stück des Weges gehen. Nur so erfährt sich der multimorbide Mensch als wertvolle, einzigartige Person, die ernst genommen wird. Nur so wird er 
nicht zum Objekt von Fürsorge- und Dienstleistungen, Erwartungen, Bewertungen, Belehrungen, Beurteilungen und Anordnungen gemacht.

\subsubsection{Aber was könnte konkret die Umsetzung einer effizienten geriatrischen primären Gewaltprävention im Sinne von Gesundheitsförderung für den geriatrischen Patienten bedeuten?}

Gesundheit kann bei alten und hochaltrigen Menschen nur im Sinne von Wohlbefinden verstanden werden. Der Begriff Wohlbefinden ist für ein biomedizinisches Paradigma ein großes Problem, da es nicht messbar, nicht objektivierbar, nicht nachweisbar ist. Wohlbefinden bedeutet konkret, dass das Erleben, die Empfindungen des sehr alten Menschen mit seiner jeweiligen Biografie, seiner Persönlichkeit, seinen Bewältigungsstrategien, seinen Gewohnheiten, Merkmalen und seinem Alltag berücksichtigt werden. Klaus Hurrelmann hat 2003 sehr treffend formuliert: „Gesundheit ist ein Stadium, das einem Menschen Woblbefinden und Lebensfreude vermit-

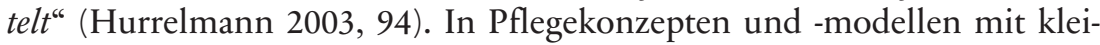
ner Reichweite sind Bereiche verankert wie Ethik, Menschenwürde und die Haltung gegenüber Menschen. Diese Modelle ermöglichen eine bessere Situationsanalyse, auf deren Grundlage erst eine situations- und individuumsangemessene Hilfeplanung erstellt und deren Zielsetzungen durchgeführt werden können. Warum ist diese situations- und individuumsangemessene Pflegeplanung so wichtig? Das Empowerment-Konzept als Stärkung von Kompetenz, Autonomie und Selbstbestimmung über die eigene Gesundheit im Rahmen der situations- und individuumsangemessenen Pflegeplanung verabschiedet sich von der Defizitorientierung und richtet den Blick auf die Selbstgestaltungskräfte. Es blickt auf die Fähigkeiten des Menschen. Eine der Fähigkeiten einer Person mit kognitiver Beeinträchtigung ist z. B. die nonverbale Kommunikationsebene. Die Aufgabe der Pflegepersonen sehe ich in der Erschließung von Möglichkeitsräumen in der Mikrowelt des Pflegealltags. Sie soll die Person mit Demenz bei dem Versuch, ihre Gefühle, ihr Erleben, ihre Sorgen und ihre Freude auszudrücken, unterstützen. Die nonverbale Kommunikation soll als Ressource der Person mit kognitiver Beeinträchtigung betrachtet werden, um Veränderungen in belastenden Ist-Situationen zu erzielen. Eine Unterstützung entsteht beispielsweise durch das aktive Zuhören, durch die Wahrnehmung der nonverbalen Signale, die die Person mit kognitiver Beeinträchtigung permanent sendet, durch Ermutigung, eigene, vielfach verschüttete nonverbale Ressourcen einzusetzen, durch Urvertrauen in die Stärken jedes Menschen, durch Unterstützung von Autonomieversuchen, wie dem Ver- 
such, allein zu essen, sich anzuziehen oder auf die Toilette zu gehen, durch Vorzeigen, wie einfache Handlungen ablaufen oder wie verschiedene Utensilien benützt werden, durch Erlauben der von den Gesellschaftsnormen abweichenden Verhaltensweisen, wie z. B. mit den Fingern essen oder das Tragen bunter Kleiderkombinationen, und durch Rhythmusanpassungen. Nur so kann sich die Person mit kognitiver Beeinträchtigung noch kompetent fühlen. Diese Aufgaben müssen die Mitarbeiter tagtäglich sehr ernst nehmen. Dabei sollte nicht vergessen werden, dass auch Humor als Interaktionskompetenz erlebbar zu gestalten ist.

\subsubsection{Was bedeutet die Umsetzung einer effizienten geriatrischen \\ Gewaltprävention im Sinne von Gesundheitsförderung für das Personal?}

Drei Grundlagen scheinen unverzichtbar zu sein:

- Entscheidungen müssen miteinander getroffen und die Mitarbeiter müssen ernst genommen werden.

- Selbstverantwortung soll großgeschrieben werden, damit Pflegepersonen das Gefühl haben, dass das, was sie tun, sinn- und wertvoll ist.

- Gute Beziehungen und die Kooperation zwischen Pflegedienstleiter und Mitarbeiter dienen als Basis für die Motivation der Mitarbeiter.

Die Aufgabe der professionellen Pflege in der Pflege von alten und hochaltrigen Menschen muss auf einer vertrauensvollen Beziehung zwischen betreuten Personen und Pflegepersonen beruhen. Dies basiert auf Empathie, Zuwendung, Einfühlungsvermögen und Anteilnahme. Diese offene Beziehung ermöglicht es, die Ressourcen beider Interaktionspartner wahrzunehmen und das Wohl beider zu entfalten. Gemeinsam suchen sie und fixieren sie konkrete kurz-, mittel- und langfristige Ziele, um die Lebensqualität beider zu unterstützen: die Lebensqualität für den Bewohner, aber auch die Lebensqualität in der Arbeit der Pflegepersonen. Gesundheitsförderliche Lebenswelten zu schaffen, bedeutet, dass die Lebenswelten stationär betreuter Menschen und die Arbeitswelt der Pflegepersonen in einem Rahmen stattfinden können, der das Wohlbefinden aller Beteiligten berücksichtigt. Die Pflegedienstleitung muss es schaffen, Rahmenbedingungen herzustellen, die physische, psychische, spirituelle, lebensweltliche sowie soziokulturelle Erfahrungen der Bewohner und der Mitarbeiter respektieren und ethische Richtlinien berücksichtigen. Gute Beziehungen am Arbeitsplatz, Gerechtigkeit und erlebtes Vertrauen wirken nicht nur motivierend, sondern wirken gewaltpräventiv und sind auch gesundheitsfördernd. Mitarbeiter, die in den Prozess der Qualitätsentwicklung eingebunden 
sind, die Selbstverantwortung tragen, fürchten sich nicht mehr vor Qualitätsevaluationen. Fachwissen und Eigenverantwortung führen dazu, dass Pflegepersonen lernen, sich zu artikulieren und zu argumentieren. Durch gemeinsame Indoor-Schulungen und eine gemeinsame Haltung sprechen die Pflegepersonen die gleiche Sprache. Das Pflegehandeln, das in den Selbstverantwortungsbereich des Pflegenden gehört, ist "State of the Art“, stets nachvollziehbar, argumentierbar und begründet. Das Ergebnis ist die Einstufung des Qualitätsniveaus der Pflege auf Stufe 4 und damit optimale Pflege: „Der Bewohner erhält die Möglichkeit, die Pflege aktiv mitzugestalten und selbst zu entscheiden, die Anforderungen entsprechen seinen Fähigkeiten“ (Kämmer 1994, 134).

Der alte Mensch wird in der Suche nach Kräften, nach eigener Wertigkeit, die ein „besseres Leben“ ermöglichen, unterstützt. Was ein „Mehr an Lebenswert" ausmacht, kann nur der alte Mensch definieren im Rahmen seiner Lebenseinstellungen, Prioritätenskalen und Grundüberzeugungen. Ein „Mehr an Lebenswert“ ist dadurch eine offene, individuelle, normative Form. Der alte Mensch wird (wieder) Regisseur der eigenen Biografie. Er wird zum Experten in eigener Sache. Partizipation und Selbständigkeit werden gefördert. Die Kontinuität kann unter anderem durch Pflegevisiten gewährleistet werden: Die ständige Kommunikation in Pflegevisiten und deren Verschriftlichung stellen sicher, dass Maßnahmen mit dem oder ohne den Pflegebedürftigen vereinbart wurden und dass alle Pflegepersonen an diesen Zielen beteiligt sind, daran arbeiten, Konzepte umsetzen und danach leben. Gemeinsame Lösungen werden gesucht, Informationen fließen in alle Richtungen. Es gibt viele kleine, temporäre Arbeitsgruppen, sei es für Trauerbegleitung, für Schmerztherapie, für Urlaub vom Heim oder für Pflegeleitbilder. Die Ergebnisse aus diesen Arbeitsgruppen fließen in die Alltagspraxis ein.

Eine wichtige Aufgabe der Pflege ist auch die Unterstützung der Angehörigen - falls sie noch vorhanden und bereit sind, Kontakt zu pflegen unter anderem durch Information über das Pflegeverständnis, den Krankheitsverlauf und den Sterbeprozess sowie durch Gesprächsrunden. Vertieftes Wissen hilft, Situationen klarer zu sehen und Varianten zur Gewalt zu analysieren. Dies soll ihnen ermöglichen, Entscheidungen im Sinne des Bewohners zu treffen (besonders, wenn sie die Sachwalter bzw. Erwachsenenvertreter sind) oder zumindest mitzutragen. 


\subsubsection{Was bedeutet die Umsetzung einer effizienten geriatrischen Gewaltprävention im Sinne von Gesundheitsförderung für die Organisation?}

Die Stärke von Führungskräften liegt in der Wirksamkeit und Ausdauer: Sie arbeiten im Hintergrund und sind ganz gewöhnliche Menschen, die konstant, wie ein Motor, das Schiff vorantreiben. Die Stärke eines Stationsleiters wird besonders sichtbar in seiner Fähigkeit, einen Mitarbeiter so einzusetzen, dass dieser sich weiterentwickeln und verwirklichen kann. Das ist eine Kunst, die von der Pflegedienstleitung vorgelebt werden muss: präsent zu sein, ohne dabei aufdringlich zu wirken; die Geschichte jedes Bewohners und jedes Mitarbeiters so gut zu kennen, dass er oder sie sich wahrgenommen und ernst genommen fühlt; zu führen, ohne zu manipulieren; Raum für Kreativität und Freiheit zu bieten, ohne Chaos entstehen zu lassen. Der Pflegedienstleiter kann mit einem Orchesterdirigenten verglichen werden. Jeder Musiker ist ein Experte. Das Bild des Orchesters wurde in anderer Form von Erich Loewy bereits in den 90er Jahren verwendet (Loewy 1995, 104). Eine Symphonie zu dirigieren oder eine Partitur zu schreiben, heißt, die richtigen Instrumente in der richtigen Tonart und zum richtigen Zeitpunkt zu verwenden. Es ist eine Aufgabe, die nicht nur technisches, sondern in erster Linie auch Wissen um menschliche Gefühle voraussetzt. Die Rolle des Orchesterdirigenten ist es, diese Solisten so zu führen, dass sie ein Musikstück auf höchstem Niveau gemeinsam spielen, ohne dabei ihre Eigenheiten zu verleugnen. Jeder Einzelne ist wichtig. Die Aufgabe des Dirigenten ist es, den Einsatz genau zu koordinieren, die Betonungen und Rhythmen aufzuzeigen, um den eigenen Klang des Orchesters entstehen zu lassen. All das geschieht zu dem Zweck, das „größere Ganze“, ein einzigartiges Musikstück eines besonderen Komponisten, hörbar und spürbar zu machen. Der Pflegedienstleiter hat nur diese eine Aufgabe - seine Musiker sind die Mitarbeiter und das größere Ganze ist das Angebot einer Betreuung und Pflege auf hohem Niveau bei gleichzeitiger Wirtschaftlichkeit. Der Pflegedienstleiter wie der Dirigent behält das ganze Orchester und seinen Rahmen im Auge. Die Gesamtverantwortung trägt die Führungskraft, aber durch Weitergabe von Verantwortung an die Mitarbeiter wird wiederum die Führungskraft von den Mitarbeitern getragen. Dieses Geben und Empfangen von Kraft ist ein entscheidendes Mittel gegen Überforderung und Burnout. Es wirkt stabilisierend auf den Ressourcenhaushalt aller Personen, die in der Organisation arbeiten, und schafft die Grundlage einer Vertrauensbasis.

Ein weiterer Aspekt dieses Gebens und Empfangens ist das Zugestehen der Möglichkeit, zu sagen: „Ich habe einen Fehler begangen“, oder: „Hier 
sind wir auf dem falschen Weg." Achtet der Pflegedienstleiter und mit ihm die Stationsleitungen auf ihre Mitarbeiter, dann dürfen sie sich entfalten. In einer Organisation, die Heiterkeit, Inspiration, Respekt und Achtsamkeit vorlebt, wird jeder Einzelne ernst genommen und braucht keine Gewalt, keine Macht auszuüben.

„Eine gute Stimmung ermöglicht erst, Mitgefühl und Wertschätzung für sich selbst und andere Menschen haben zu können. Erfährt man den Wert, den man sich wünscht, kann man auch den Mitmenschen Wertschätzung entgegenbringen." (Fraberger 2014, 235)

Jeder Mitarbeiter hat das Recht, er selbst zu sein. Der Pflegedienstleiter einer Organisation stellt nicht wahllos jemanden an, weil gerade irgendwo jemand gebraucht wird, sondern er besetzt jeden einzelnen Posten ganz gezielt: z. B. Frau Konrad, weil sie Frau Konrad ist und über bestimmte Kompetenzen, Fertigkeiten und Fähigkeiten verfügt. Empowerment meint hier einen Prozess, innerhalb dessen Pflegepersonen lernen, ihre eigene Arbeitswelt, ihre soziale Umwelt in die Hand zu nehmen. Sie begeben sich auf die Suche nach eigenen Kompetenzen, Ressourcen und Kräften. Sie trauen sich, ihre selbst erarbeiteten Lösungsansätze zu präsentieren, zu argumentieren und zu leben. Dafür benötigen sie aber Vorbilder.

\section{Fazit}

Projekte zur Gewaltprävention und damit zur Verbesserung der Betreuungsqualität von alten und hochaltrigen Menschen im intramuralen Bereich können sich sicher nicht nur auf die Weiterbildung des Betreuungsteams beschränken. Es wäre zu einseitig und zum Scheitern verurteilt. Die Umsetzung des Empowerment-Konzeptes erfordert einen Paradigmenwechsel im institutionellen Selbstverständnis, im Organisationsleitbild, im Bild des alten Menschen, im Methodenkatalog, im Problemlösungsverfahren, in der Kultur des Helfens. Eine Umsetzung der Patientorientierung ist mit einer Wahrnehmung der Bedürfnisse des Pflegepersonals verbunden. Nur wenn beide Interaktionspartner sich wohlfühlen, kann es Lebensqualität geben.

Zusammenfassend können wir mit Secretan einen Grundsatz der Gewaltprävention aussprechen: „Wir dürfen keine Verhaltensweisen tolerieren, die das Selbstwertgefühl der Menschen, ihre Hoffnungen, ihre Individualität oder ihre Würde untergraben.“ (Secretan 1997, 292) 


\section{Literatur}

Baker, Phlipp Robert/Francis, Daniel Peter/Hairi, Noran Naqiah Mohd/Othman, Sajaratulnisah/Choo, Wan Yuen: Interventions for preventing abuse in the elderly, in: https://www.cochranelibrary.com/cdsr/doi/ 10.1002/14651858.CD010321.pub2/full [09.11.2018].

Bauer Thomas: Kommunikation wissenschaftlich denken. Perspektiven einer kontextuellen Theorie gesellschaftlicher Verständigung, Wien usw.: Böhlau 2014.

Bundesgesetzblatt für die Republik Österreich. 69. Bundesgesetz: Änderung des Bundesgesetzes über Krankenanstalten und Kuranstalten, in: https://www.ris.bk a.gv.at/Dokumente/BgblAuth/BGBLA_2011_I_69/BGBLA_2011_I_69.pdfsig [09.11.2018].

Cannell, Brad M./Jetelina, Katelyn K./Zavadsky, Matt/Reingle Gonzales, Jennifer M.: Towards the development of a screening tool to enhance the detection of elder abuse and neglect by emergency medical technicians (EMTs): a qualitative study, in: BioMed Central (BMC) Emergency Medicine 16/19 (2016).

Cooper, Claudia/Selwood, Amber/Livingston, Gill: The prevalence of elder abuse and neglect: a systematic review, in: Age and Ageing 37 (2008) 151-160.

Council of Europe: Übereinkommen des Europarates zur Verhütung und Bekämpfung von Gewalt gegen Frauen und häuslicher Gewalt. Istanbul-Konvention vom 11.05.2011, in: https://www.interventionsstelle-wien.at/istanbul-konventio n [09.11.2018].

Dieck, Margret: Gewalt gegen ältere Menschen im familialen Kontext - Ein Thema der Forschung, der Praxis und der öffentlichen Information, in: Zeitschrift für Gerontologie (ZfG) 20 (1987) 305-313.

Europäische Menschenrechtskonvention (EMRK), in: https://www.menschenrechts konvention.eu/ [09.11.2018].

Fraberger, Georg: Ein ziemlich gutes Leben, Salzburg: Ecowin 2014.

Fried, Erich: Die Gewalt, in: Ders.: Um Klarheit, Berlin: K. Wagenbach 1985.

Frühwald, Thomas: Diagnose Gewalt. Gewalt aus der Sicht der Geriatrie. Drittes FSW-ExpertInnen-Forum „Gewalt gegen Alte“ am 18. November 2008 in Wien. Vortragsmanuskript.

Galtung, Johan: Konflikte und Konfliktlösungen. Die Transcent-Methode und ihre Anwendung, Berlin: Kai Homilius 2007 (= Globale Analysen 3).

Galtung, Johan: Kulturelle Gewalt, in: Der Bürger im Staat 43/2 (1993) 106-112.

Galtung, Johan: Strukturelle Gewalt. Beiträge zur Friedensforschung, Reinbek bei Hamburg: Rowohlt 1975.

Görgen, Thomas/Herbst, Sandra/Kotlenga, Sandra/Nägele, Barbara/Rabold, Susann: Kriminalitäts- und Gewalterfahrungen im Leben älterer Menschen. Zusammenfassung wesentlicher Ergebnisse einer Studie zu Gefährdungen älterer und pflegebedürftiger Menschen, Berlin: Bundesamt für Familie, Senioren, Frauen und Jugend 2012. 
Hantikainen, Virpi: Freiheitsbeschränkende Maßnahmen in der Pflege betagter Menschen. Drittes FSW-ExpertInnen-Forum „Gewalt gegen Alte“ am 18. November 2008 in Wien. Vortragsmanuskript.

Hirsch, Rolf Dieter: Gewalt gegen alte Menschen. Erkennen - Sensibilisieren Handeln!, in: https://link.springer.com/article/10.1007\%2Fs00103-015-2268-5\#et hics [09.03.2020].

Hirsch, Rolf Dieter: Gewalt und Misshandlungen in Pflegeinrichtungen und deren Prävention, in: Deutsches Institut für Menschenrechte (Hg.): Prävention von Folter und Misshandlung in Deutschland, Baden-Baden: Nomos 2007.

Hirsch, Rolf Dieter: Prävention und Intervention gegen Gewalt bei alten Menschen in Einrichtungen, in: http:/www.humanrights.ch/home/upload/pdf/0508 25_hirsch_praevention.pdf [08.11.2018].

Hörl, Josef: Gewalt hat viele Gesichter und Namen, in: Humer, Brigitte (Hg.): Gewalt an alten Menschen. Erkennen - Informieren - Verhindern, Linz: Pro Mente Edition 2014 (= Schriften zur Sozialen Arbeit 27).

Hüther, Gerald: Raus aus der Demenz-Falle! Wie es gelingen kann, die Selbstheilungskräfte des Gehirns rechtzeitig zu aktivieren, München: Arkana 2014.

Hurrelmann, Klaus: Gesundheitssoziologie. Eine Einführung in sozialwissenschaftliche Theorien von Krankheitsprävention und Gesundheitsförderung, Weinheim: Juventa 2003.

Jackson, Selly L./Hafemeister, Thomas L.: APS investigation across four types of elder maltreatment, in: Journal of Adult Protection 14/2 (2012) 82-92.

Kämmer, Karla (Hg.): Pflegemanagement in Altenheimen. Grundlagen für Konzeptentwicklung und Organisation, Hannover: Schlütersche Verlagsanstalt 1994.

Kruse, Andreas: Demenz - medizinisch-pflegerische, psychologische, ethische Überlegungen, in: Kirchenamt der EKD (Hg.): Leben mit Demenz. Beiträge aus medizinisch-pflegerischer, theologischer und lebenspraktischer Sicht, Hannover: EKD 2008 (= EKD-Texte 98), 45-79.

Lachs, Mark/Pillemer, Karl: Elder Abuse, in: The New England Journal of Medicine 373/20 (2015) 1947-1956.

Lachs, Mark/Williams, Christianna/O’Brien, Shelley/Pillemer, Karl/Charlson, Mary: The mortality of elder mistreatment, in: Journal of the American Medical Association 280 (1998) 428-432.

Loewy, Erich H.: Ethische Fragen in der Medizin, Wien und New York: Springer 1995.

Maio, Giovanni: Geschäftsmodell Gesundheit. Wie der Markt die Heilkunst abschafft, Berlin: Surkamp 2016 (= medizinHuman 15).

Nachbaur, Dina: GEWALTige Herausforderungen in allen Lebenslagen, in: Berzlanovich, Andrea/Brinek, Gertrude/Rösslhumer, Maria (Hg.): Eine von Fünf. Gewaltschutz für Frauen in allen Lebenslagen, Wien und Saarbrücken: Edition Ausblick 2017, 38-44. 
Ogris, Günther: Gewalt an Frauen - die soziologische Perspektive, in: Berzlanovich, Andrea/Brinek, Gertrude/Rösslhumer, Maria (Hg.): Eine von Fünf. Gewaltschutz für Frauen in allen Lebenslagen, Wien und Saarbrücken: Edition Ausblick 2017, 14-19.

Pflegerl, Johannes/Cizek, Brigitte: Problemstellungen der Forschung, in: Bundesministerium für soziale Sicherheit und Generationen (Hg.): Gewalt in der Familie - Rückblick und neue Herausforderungen. Gewaltbericht 2001, Wien: 2002, 56-68.

Popitz, Heinrich: Phänomene der Macht, Tübingen: Mohr 1992.

Schiffczyk, Claudia/Romero, Barbara/Jonas, Christina/Lahmeyer, Constanze/ Müller, Friedemann/Riepe, Matthias W.: Appraising the need for care in Alzheimer's disease, in: BioMedCentral (BMC) Psychiatry (2013) 13-73.

Secretan, Lance: Soul-Management. Der neue Geist des Erfolgs - die Unternehmenskultur der Zukunft, München: Lichtenberg 1997.

Sozialministerium: Sozialstaat Österreich. Leistungen, Ausgaben und Finanzierung 2016, in: https://broschuerenservice.sozialministerium.at/Home/Download?publ icationId=336 [09.11.2018].

Traxler, Christine: Nursing Home Abuse Center Protecting Our Seniors: Elder Abuse Statistics, in: https://www.nursinghomeabusecenter.com/elder-abuse/ statistics/ [09.11.2018].

Weissenberger-Leduc, Monique/Weiberg, Anja: Gewalt und Demenz. Ursachen und Lösungsansätze für ein Tabuthema in der Pflege, Wien: Springer 2011.

WHO/Alzheimer's Disease International: Dementia. A Public Health Priority, in: http://www.who.int/mental_health/neurology/dementia/dementia_thematicbrief_executivesummary.pdf [09.11.2018].

WHO: European report on preventing elder maltreatment, in: http:// www.who.int/iris/handle/10665/107293 [08.11.2018].

WHO: Toronto Declaration on the Global Prevention of Elder Abuse, in: http:// www.who.int/ageing/projects/elder_abuse/alc_toronto_declaration_en.pdf [09.11.2018]. 
